\title{
Uncertainty of slip measurements in a cutting system of converting machinery for diapers production
}

\author{
F. D'Aponte ${ }^{1}$, G. D’Emilia ${ }^{2}$, S. Lupinetti ${ }^{1}$, E. Natale $^{2, \star}$, and P. Pasqualoni ${ }^{1}$ \\ 1 Fameccanica Data Spa, Via Aterno, 136, Zona Industriale Val Pescara, Chieti, Italy \\ 2 Department of Industrial and Information Engineering and Economics, University of L'Aquila, via G. Gronchi 18 \\ 67100 L'Aquila, Italy
}

Received: 7 May 2015 / Accepted: 6 July 2015

\begin{abstract}
In this paper slip measurements are described between the peripheral surfaces of knife and a not driven anvil cylinders in a high velocity, high quality cutting unit of a diaper production line. Laboratory tests have been carried out on a test bench with real scale components for possible on line application of the method. With reference to both starting and steady state conditions correlations with the process parameters have been found, achieving a very satisfactory reduction of the slip between the knife cylinder and the not driven anvil one. Accuracy evaluation of measurements allowed us to validate the obtained information and to evaluate the detection threshold of the measurement method in the present configuration The analysis of specific uncertainty contributions to the whole uncertainty could be also used, to further reduce the requested uncertainty of the measurement method.
\end{abstract}

Keywords: Cutting of film, measurement uncertainty, nonwoven textile, process monitoring, slip

\section{Nomenclature}

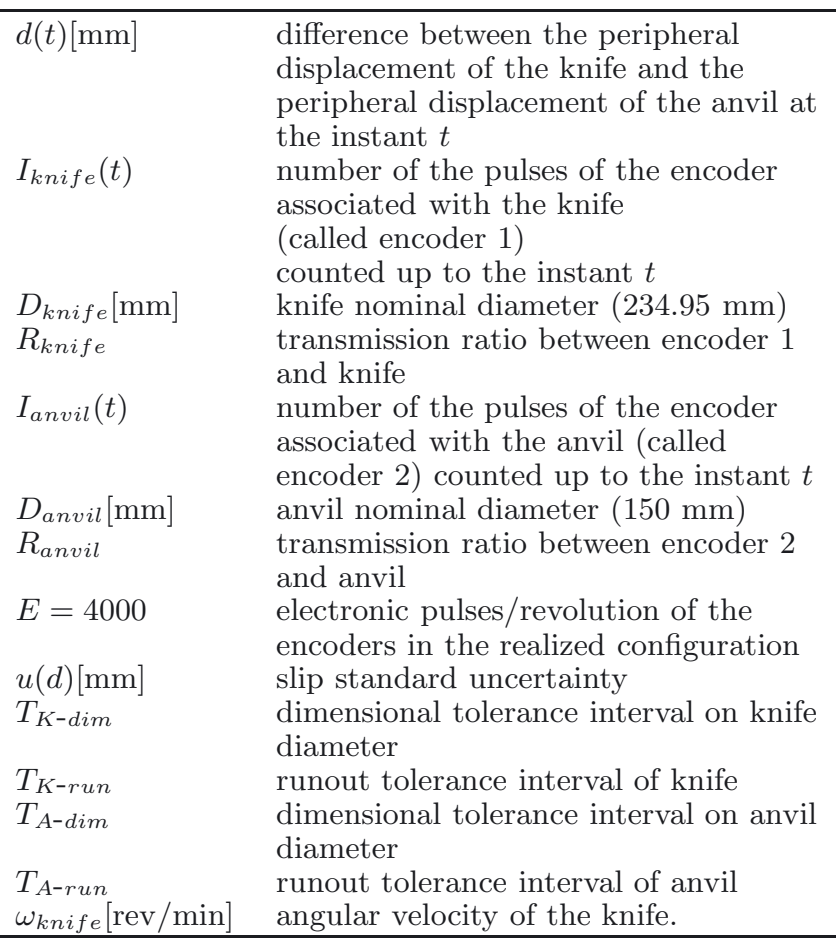

^ Correspondence: emanuela.natale@univaq.it

\section{Introduction}

Many industrial applications still exist where the contemporaneous need of increase of product quality and of production rate is necessary. Obviously, the contemporaneous achievement of these requirements asks for actions on many aspects concerning both the design of components of the production line and the control of the production process.

Among many examples that could be indicated, an interesting application refers to nonwoven textile industry: in fact, if the specifications of these automatic production lines are considered, the increase of production rate and product quality is requested, for both economic and commercial reasons and with reference to traditional and emerging world markets together.

In this paper, the attention is focused on converting machinery for diapers production, for both technical complexity of the production line and its economic relevance, if it is considered on the whole.

In fact, the increase of technical performances of products, the reduction of raw materials and energy consumption require that improvements in many production phases are carried out.

Among many aspects, the cutting of pieces of materials to be assembled is an operating phase of remarkable importance [1-3].

In fact, a clean and accurate cut allows us to realize diapers, which are geometrically and esthetically 
appreciable; on the other hand, a cut correctly operated from a mechanical point of view, with reference to both the contact forces between knife and anvil and the relative motion between them, is able to cooperate to a good cutting action and to reduce the wear of knives and the production of fibers. These actions allow us to improve the stability of performances during the time, so reducing the maintenance actions and cost.

Taking into account the above considerations, the cutting operation has been deeply studied, with reference to the characteristics of nonwoven web film and of the kinematic conditions existing between knife and anvil; there are also examples of cutting tools specifically realized and instrumented to monitor the cutting process for both quality improvement of product and wear reduction of the cutting tool. Many interesting information can be obtained, even though the proposed solutions appear difficult to be implemented directly on line.

In fact, in literature many geometrical and dynamical parameters are studied, like the cutting edge height [4] and width [5], the kinematic radial coupling of knife and anvil [6], the wear of cutting tools [7], the contact force [8]. However, no attention is paid to the relative peripheral slip between the cylinder carrying the knives and the anvil cylinder and to the operating parameters influencing it, like contact pressure; reducing the slip is useful in order to improve the cutting quality and an improvement of geometrical specification of pieces could be finally achieved.

In this paper, an experimental research is described, concerning the evaluation of the relative slip between the surfaces of knife and anvil cylinders in the cutting stage of a high speed, high quality production line of diapers; the effect of the force acting to bring together knife and anvil is also carefully analyzed in order to verify the possibility of realizing good working conditions of the cutting unit, with specific and careful attention to the slip between knife and anvil. The acting force and, consequently, the pressure of the pneumatic system, that pushes the anvil against the knife, have been taken into account, because the pressure is the only operating parameter acting specifically on the cutting unit. In fact, design aspects of the cutting unit (number of knives, height and thickness of knives, ...) are defined in the design phase of the system; the design guide lines are mainly based on production requirements and they are not taken into consideration in this work.

The proposed slip measurement system is based on simple solutions, even though carefully studied in literature [9-13], in order to measure the quantities of interest with the aim of also checking the possibility of implementing them directly on line.

Moreover, particular attention is paid to the uncertainty of measurement, taking into account all the effects that are relevant in the process.

Uncertainty evaluation is useful to both accurately evaluate the slip and to individuate the limits of the measurement method, in particular with respect to its ability of individuating the change of working conditions of the cutting unit, also for monitoring and diagnosing purposes.

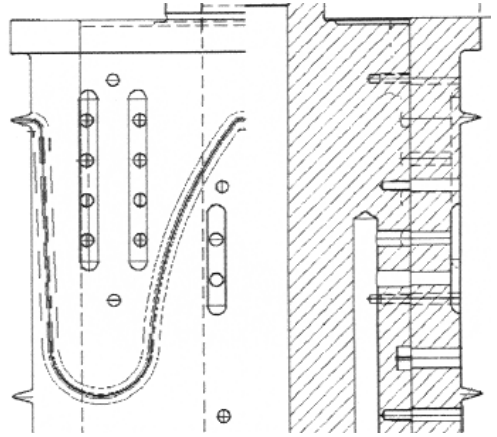

a)

elastic force applied to the anvil shaft

b)

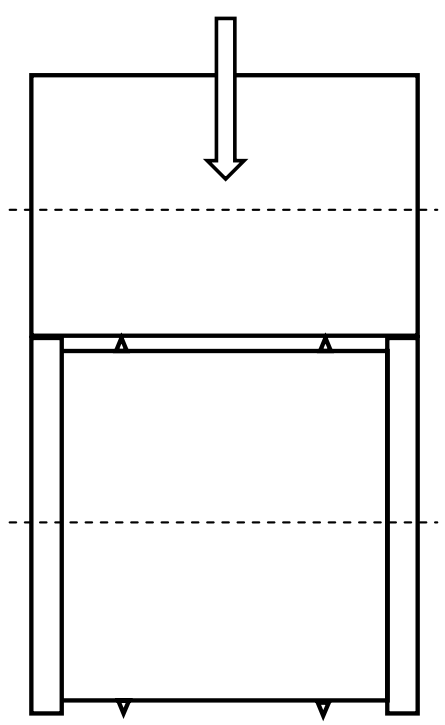

Fig. 1. (a) Drawing of the knife; (b) scheme of the knife-anvil system.

\section{Materials and methodology}

The real configuration of a cutting system of a converting machinery for diapers production has been reproduced in laboratory.

The test bench includes a revolving knife and an anvil. The former is constituted by a cylinder with sharp profiles (Fig. 1a) and the latter consists of a non-driven roller supported in a lubricated cradle, that exerts an elastic force against the cutting unit by a pneumatic system, whose pressure can be set as required (Fig. 1b). In Figure 2 a 3D drawing of the knife-anvil system is shown.

The axis of the knife is connected by a toothed belt transmission to an encoder, (encoder 1 in Fig. 3), and, similarly, the axis of the anvil is synchronously connected to an encoder (the encoder 2 in the same figure).

The synchronous transmission has been set in order to realize a better lay-out and to improve the measuring performances of the angular velocities using suitable transmission ratios between the shafts of knife and anvil and of encoder 1 and encoder 2, respectively. 


$$
u(d)=\frac{\pi}{E} \sqrt{\begin{array}{l}
\left(\frac{D_{\text {knife }}}{R_{\text {knife }}} u\left(I_{\text {knife }}\right)\right)^{2}+\left(\frac{I_{\text {knife }}}{R_{\text {knife }}} u\left(D_{\text {knife }}\right)\right)^{2}+\left(\frac{I_{\text {knife }} D_{\text {knife }}}{R_{k n i f e}^{2}} u\left(R_{\text {knife }}\right)\right)^{2} \\
+\left(\frac{D_{\text {anvil }}}{R_{\text {anvil }}} u\left(I_{\text {anvil }}\right)\right)^{2}+\left(\frac{I_{\text {anvil }}}{R_{\text {anvil }}} u\left(D_{\text {anvil }}\right)\right)^{2}+\left(\frac{I_{\text {anvil }} D_{\text {anvil }}}{R_{\text {anvil }}^{2}} u\left(R_{\text {anvil }}\right)\right)^{2}
\end{array}}
$$

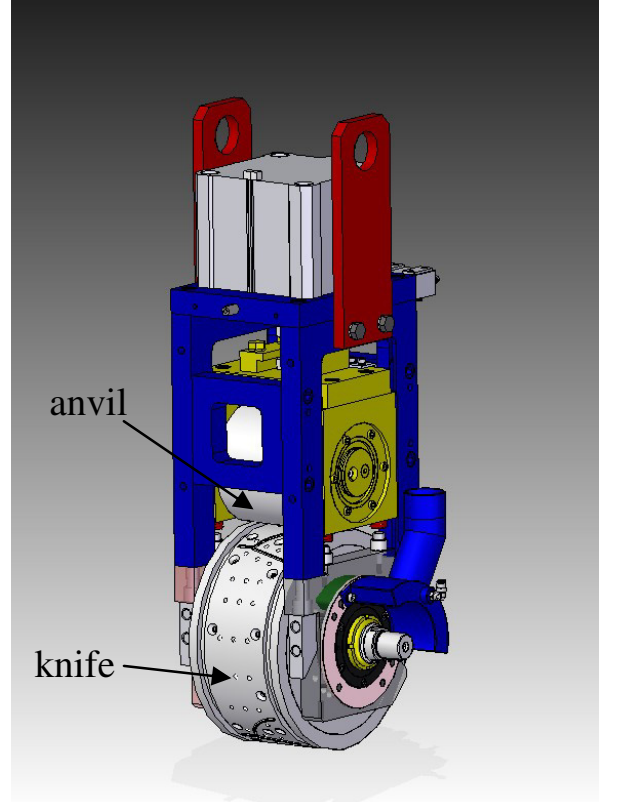

Fig. 2. 3D drawing of the knife-anvil system.

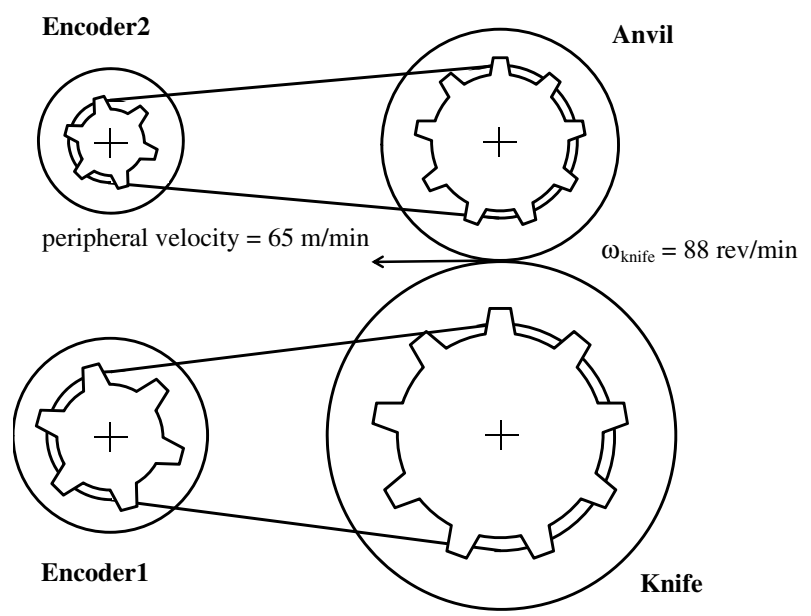

Fig. 3. Scheme of the connections between knife and encoder1, and between anvil and encoder2. Peripheral slip on the contact line depends on time.

The slip between knife and anvil is calculated by measuring the angular displacements provided by the encoders and then taking into account respective diameters.

Two incremental encoders LEINE \& LINDE, SRD 30, with a resolution of 1000 pulses/revolution realize the measurement of the angular displacements of knife and anvil.
In order to improve the accuracy of peripheral displacement measurement, the resolution has been increased to 4000 pulses/revolution, by using the two outputs out of phase of each encoder and comparing them; this specific approach allows us to obtain four on/off electronic pulses for each optical slot.

A NI CompactRIO 9104 system, based on a FPGA (Field-Programmable Gate Array), and the NI 9411 differential digital input module are used for the acquisition of the encoder signals.

The slip between knife and anvil, that is the difference between the respective peripheral displacements, is in accordance with the following formula:

$$
\begin{aligned}
d(t) & =\frac{I_{k n i f e}(t) 2 \pi}{E R_{k n i f e}} \frac{D_{\text {knife }}}{2}-\frac{I_{\text {anvil }}(t) 2 \pi}{E R_{\text {anvil }}} \frac{D_{\text {anvil }}}{2} \\
& =\frac{\pi}{E}\left(\frac{I_{k n i f e}(t) D_{\text {knife }}}{R_{\text {knife }}}-\frac{I_{\text {anvil }}(t) D_{\text {anvil }}}{R_{\text {anvil }}}\right) .
\end{aligned}
$$

The calculation of the slip is affected by uncertainty; different contributions can be individuated, related to the variables involved, namely:

- Uncertainty of the knife diameter.

- Uncertainty of the anvil diameter.

- Uncertainty of resolution of the encoders.

- Uncertainty of the transmission ratios.

The overall slip measurement uncertainty has been estimated using the following formula, which is an application of the law of the uncertainty propagation [14]:

$$
\text { see equation (2) above. }
$$

The uncertainty of each variable has been indicated by $u$, being $u$ the standard uncertainty.

The tests, carried out on the experimental bench, will be used in order to evaluate the parameters of interest:

- The slip between knife and anvil [mm].

- The relative slip (ratio of the measured slip to the peripheral advancement of the knife) between knife and anvil.

- The corresponding uncertainty.

The tests are according the following operating conditions:

- regime conditions; regime rotation velocity of the knife: $\omega_{k n i f e}=88 \mathrm{rev} / \mathrm{min}$; pressure of the pneumatic actuator: 1 bar, 1.1 bar, 1.2 bar, 1.3 bar, 1.5 bar, 1.6 bar, 2 bar, 3 bar, 4 bar, 5 bar, 6 bar.

- starting phase; regime rotation velocity of the knife: $\omega_{k n i f e}=88 \mathrm{rev} / \mathrm{min}$; pressure of the pneumatic actuator: 1 bar, 1.3 bar, 1.6 bar, 2 bar, 3 bar, 4 bar, 5 bar, 6 bar. 


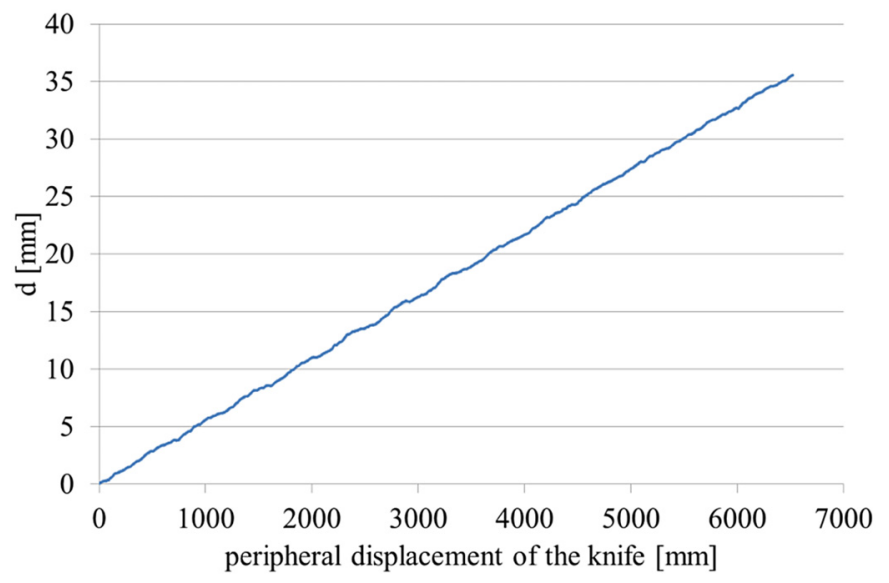

Fig. 4. Difference (d) between the peripheral displacement of the knife and the anvil one, as a function of the advancement of the knife. The curve is obtained as a mean of 10 different acquisitions.

It is to be pointed out that, even though the set measurement configuration allows to continuously evaluate the displacement, most of the presented results and discussions refer to the trend evaluation of the slip, as a global indicator of the real coupling situation.

\section{Results}

Reference operating conditions, which are considered "normal" in practice, have been defined, with the pressure of the pneumatic actuator equal to 4 bar; the reference speed is the maximum speed allowed by the system, which is about $88 \mathrm{rev} / \mathrm{min}$; the acquisition time has been set at $6 \mathrm{~s}$.

The graph of Figure 4 represents the difference between the peripheral advancement of the knife and the peripheral advancement of the anvil, as a function of the advancement of the knife. The curve is the mean of 10 different acquisitions in repeatability conditions. It is to be pointed out that the results refer to cylinders moving at a constant revolution speed, in steady conditions.

The measured slip between the anvil and the knife is in the order of the $0.5 \%$ of the peripheral advancement of the knife and this result has been considered satisfactory, with respect to the correctness of coupling between knife and anvil for cutting purposes.

The uncertainty evaluation has been carried out according to equation (2).

The contribution of the uncertainty linked to the belt transmission, which depends on factors such as the belt pitch uncertainty, non-coincidence of the primitives of belt and toothed wheels, has been evaluated under $0.5^{\circ}[12,13]$; this angle uncertainty corresponds to $1.0 \mathrm{~mm}$ of peripheral displacement of the knife, and $0.65 \mathrm{~mm}$ of peripheral displacement of the anvil; it is to be noticed that they have to be intended as overestimated contributions.

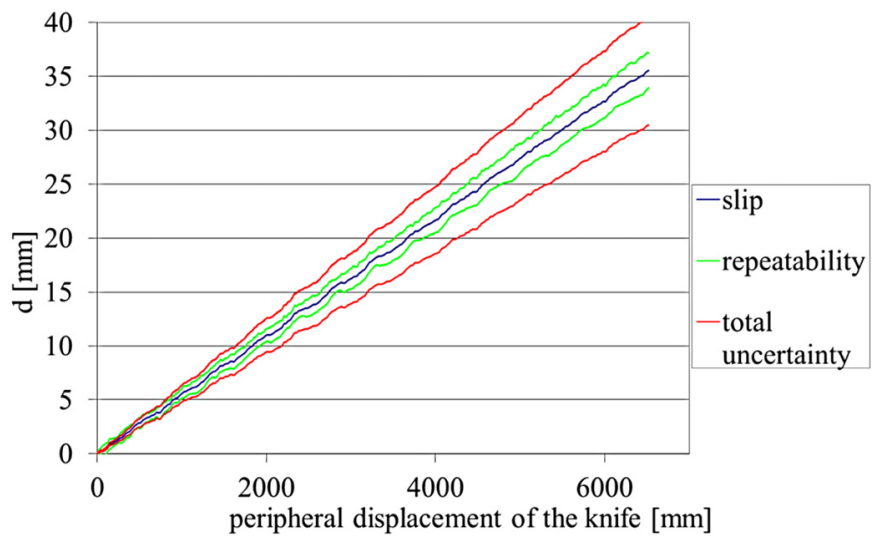

Fig. 5. Repeatability and total uncertainty of the slip measurement. See color figure at www.metrology-journal.org.

The uncertainty contributes in equation (2) have been evaluating according to the following formulas [14]:

$$
\begin{aligned}
& u\left(D_{\text {knife }}\right)=\sqrt{\left(\frac{T_{K-\text { dim }}}{2 \sqrt{3}}\right)^{2}+\left(\frac{T_{K_{\text {run }}}}{2 \sqrt{3}}\right)^{2}}[\mathrm{~mm}] \\
& u\left(D_{\text {anvil }}\right)=\sqrt{\left(\frac{T_{C-\text { dim }}}{2 \sqrt{3}}\right)^{2}+\left(\frac{T_{C_{\text {run }}}}{2 \sqrt{3}}\right)^{2}}[\mathrm{~mm}] \\
& u\left(I_{\text {knife }}\right)=u\left(I_{\text {anvil }}\right)=\frac{1}{2 \sqrt{3}} .
\end{aligned}
$$

According to the technical drawings of this specific application, tolerance intervals are:

$$
\begin{aligned}
& -T_{K \text {-dim }}=0.1 \mathrm{~mm} . \\
& -T_{K \text {-run }}=0.003 \mathrm{~mm} . \\
& -T_{A-\text { dim }}=0.4 \mathrm{~mm} . \\
& -T_{A-\text { run }}=0.005 \mathrm{~mm} .
\end{aligned}
$$

The result of the uncertainty evaluation is depicted in Figure 5. In this figure the repeatability range is also represented, calculated as a standard deviation of 10 acquisitions.

On the basis of the obtained results a whole percentage uncertainty of approximately $0.1 \%$, with respect to the peripheral displacement of cylinder carrying the knife can be estimated; this value can be also considered as the detection limit of the method, corresponding to the minimum detectable slip, as a reliable indications, if uncertainties of all parameters are taken into account.

In order to analyze the most influent causes of uncertainty, and therefore the contributions to foremost consider in order to reduce the overall uncertainty, the effect of the uncertainty of each parameter has been described in Figure 6.

In Figure $6, c 1, c 2, c 3, c 4, c 5, c 6$ are represented, being the uncertainty contributions depending on $u\left(I_{k n i f e}\right)$, 


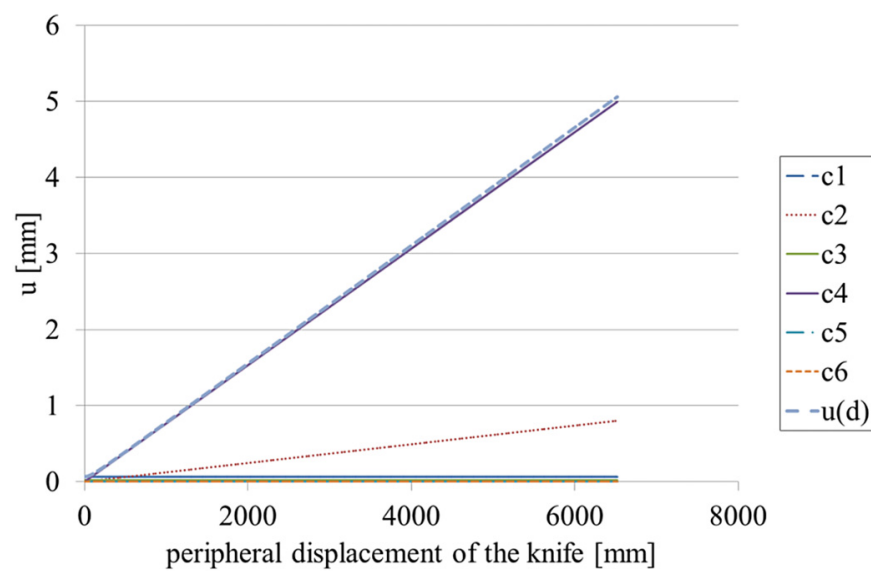

Fig. 6. Contributions of each parameter to the whole slip measurement uncertainty. See color figure at www.metrologyjournal.org.

$u\left(D_{\text {knife }}\right), u\left(I_{\text {anvil }}\right), u\left(D_{\text {anvil }}\right), u\left(R_{\text {knife }}\right), u\left(R_{\text {anvil }}\right)$, respectively, i.e.:

$$
\begin{aligned}
& c 1=\frac{\pi}{E} \frac{D_{\text {knife }}}{R_{\text {knife }}} u\left(I_{\text {knife }}\right) \\
& c 2=\frac{\pi}{E} \frac{I_{\text {knife }}}{R_{\text {knife }}} u\left(D_{\text {knife }}\right) \\
& c 3=\frac{\pi}{E} \frac{D_{\text {anvil }}}{R_{\text {anvil }}} u\left(I_{\text {anvil }}\right) \\
& c 4=\frac{\pi}{E} \frac{I_{\text {anvil }}}{R_{\text {anvil }}} u\left(D_{\text {anvil }}\right) \\
& c 5=\frac{\pi}{E} \frac{I_{\text {knife }} D_{\text {knife }}}{R_{\text {knife }}^{2}} u\left(R_{\text {knife }}\right) \\
& c 6=\frac{\pi}{E} \frac{I_{\text {anvil }} D_{\text {anvil }}}{R_{\text {anvil }}^{2}} u\left(R_{\text {anvil }}\right)
\end{aligned}
$$

All the $c 1$ to $c 6$ contributions are in millimeters.

According to previous evaluations, constant values have been assumed for $c 5$ and $c 6$, equal, respectively, to $1.0 \mathrm{~mm}$ and $0.65 \mathrm{~mm}$. In fact, since the transmission with toothed belts is synchronous, the ratios $R_{k n i f e}$ and $R_{\text {anvil }}$ have negligible uncertainty if an integer number of cycles is considered; within every cycle the maximum uncertainty contributions are $1.0 \mathrm{~mm}$ and $0.65 \mathrm{~mm}$, as previously discussed.

The most important contributions are related to the uncertainty of diameters, in particular of the anvil one, which presents larger dimensional tolerance with respect to the knife. The diameters uncertainties depend on the manufacture quality and on the dimensional class of diameters. The dimensional classes of knife and anvil are set depending on the production requirements, and they don't take into account the reduction of the slip uncertainty. As a consequence, the measurement uncertainty is related to the specific application and it should be estimated according to equation (2).

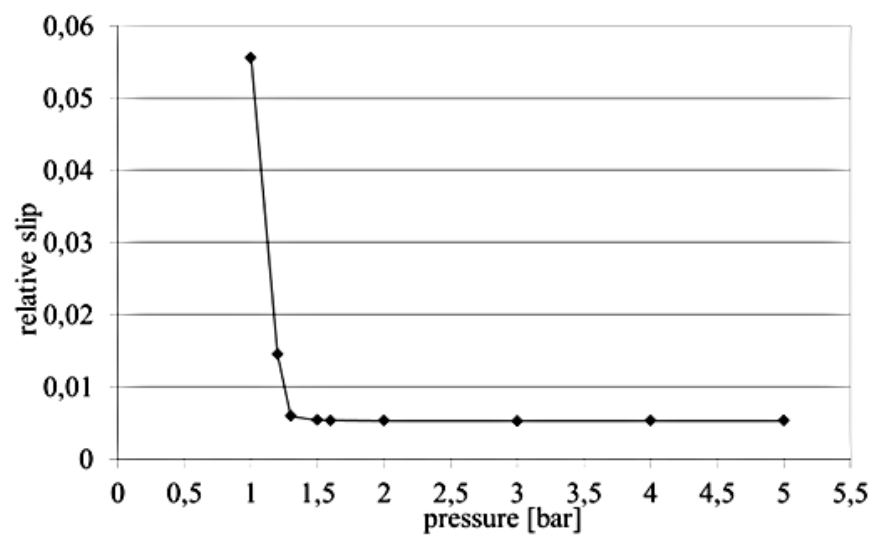

Fig. 7. Mean values of the relative slip in different pressure conditions.

To obtain a further check of the estimated uncertainty contributions and to verify the effect of working conditions on the slip, tests for slip measurements have also been carried out under different pressure conditions of the pneumatic system, that pushes the anvil against the knife.

Curves in Figure 7 are the average values of the relative slip (ratio of the measured slip to the peripheral advancement of the knife) obtained in repeated tests at pressures ranging from 1 bar up to 6 bars. Particular attention was paid to the pressure interval up to 2 bar.

The graph shows that relative sliding is maintained substantially constant and equal to a minimum value of 0.005 beyond 1.6 bar, whereas for pressure values lower than 1.6 bar, the slip increases; nominal diameters have been assumed for the slip evaluation, the effect of diameter irregularity is taken into account according to the shape tolerance limits.

In the former situation, good coupling conditions between knife and anvil are realized.

Moreover, the variability of slip measurements depends on the pressure; a repeatability of $1 \%$ has been calculated for pressure over 1.6 bar; for pushing pressure values less than that value the repeatability variability increases significantly.

These results suggest that the pressure of the pneumatic system to assure a reduced slip could be also less than 4.0 bar.

Tests show that the relative measured slip is constant and extremely repeatable in good coupling conditions.

Theoretical and experimental indications confirm that a significant reduction of uncertainty of measurement could be reached by improving the diameter measurements of specific pieces, but this could be useful only if special requirements of accuracy are necessary.

As a further information regarding the regularity of working of the cutting unit, the slip in the starting phase has been also evaluated, at different pressures from 1 to 6 bar, and the results are summarized in the graphs of Figures 8 and 9 .

If the pneumatic pressure is less than 3 bar, a significant slip occurs at the start, being negligible at higher pressures; furthermore, the slip behavior in steady state 


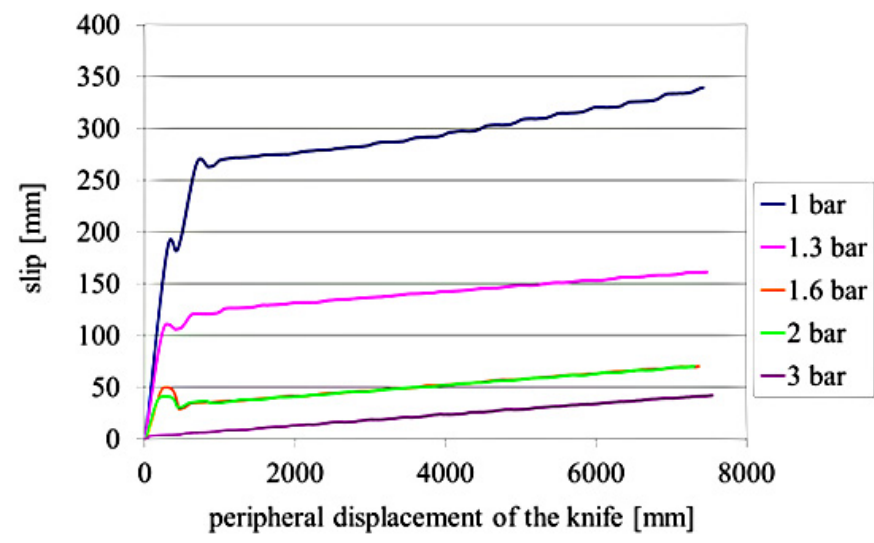

Fig. 8. Slip in the starting phase vs peripheral displacement of the knife, in different conditions of pressure (1-3 bar). See color figure at www.metrology-journal.org.

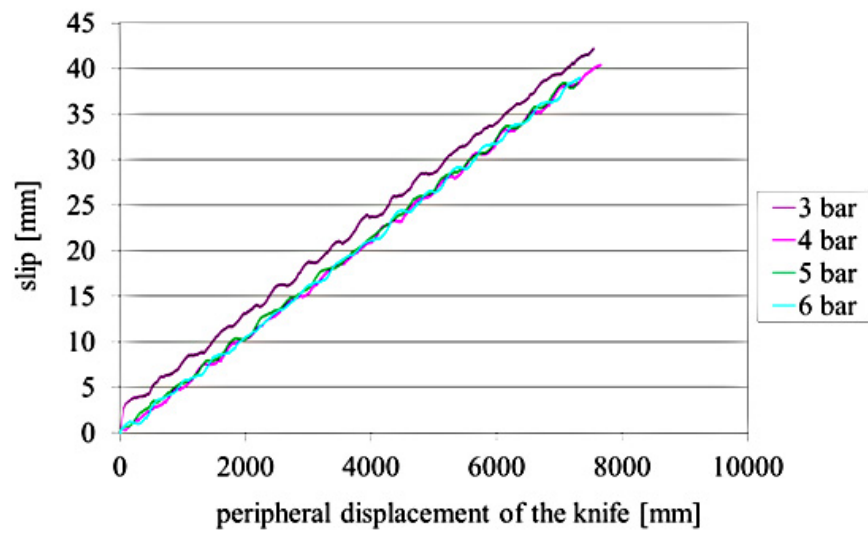

Fig. 9. Slip in the starting phase vs peripheral displacement of the knife, in different conditions of pressure (3-6 bar). See color figure at www.metrology-journal.org.

conditions is similar to previous tests; the repeatability of results also is independent on the pressure.

\section{Conclusions}

In this paper experimental results have been described, concerning the measurement of slip between a knife and an anvil cylinder of a cutting unit of a high speed, high quality automatic production line of diapers. Useful information on the pressure to be set in the pneumatic system realizing the elastic force pushing the anvil against the cutting unit has been obtained. Choosing a right pressure allows to remarkably reduce the relative slip both a the starting time and in steady state conditions.

If the slip is considered, satisfactory working conditions have been realized also at a reduced pressure with respect to "usual" settings.

Particular attention has been paid to the uncertainty of measurements, which allowed us to realize accurate measurements of slip also with a simple and inexpensive configurations, suitable for on line application. A comparative evaluation of uncertainty contribution of different quantities, both geometrical and/or kinematic, has been carried out; it allowed us to also identify the improvements in the measurement procedure to be implemented if whole uncertainty should be reduced; reducing measurement uncertainty and, consequently, detection threshold of the measurement method could be useful to improve the monitoring and diagnostic capability of the described measurement method.

\section{References}

1. C. Arcona, T.A. Dow, The role of knife sharpness in the slitting of plastic films, J. Mater. Sci. 31, 1327-1334 (1996)

2. R.R. Meehan, J. Kumar, M. Earl, E. Svenson, S.J. Burns, The role of blade sharpness in cutting instabilities of polyethylene terephthalate, J. Mater. Sci. Lett. 18, 93-95 (1999)

3. G.A. Reilly, B.A.O. McCormack, D. Taylor, Cutting sharpness measurement: a critical review, J. Mater. Process. Technol. 153-154, 261-267 (2004)

4. Shinichi Osawa, So Ito, Yuki Shimizu, SungHo Jang, Wei Gao, Tsutomu Fukuda, Akira Kato, Kouji Kubota, Cutting edge height measurement of a rotary cutting tool by a laser displacement sensor, J. Adv. Mech. Des., Syst. Manufact. 6, 815-828 (2012)

5. So Ito, Sho Sekine, Yuki Shimizu, Wei Gao, Tsutomu Fukuda, Akira Kato, Kouji Kubota, Measurement of cutting edge width of a rotary cutting tool by using a laser displacement sensor, Int. J. Auto. Tech. 8, 28-33 (2014)

6. R.R. Meehan, S.J. Burns, Mechanics of Slitting and Cutting Webs, Exp. Mech. 38, 103-109 (1997)

7. H. Lüthje, R. Bandorf, S. Biehl, B. Stint, Thin film sensor for wear detection of cutting tools, Sens. Actuat. A 116, 133-136 (2004)

8. Chao Wang, Kai Cheng, Richard Rakowski, Xun Chen, Mingyi Cheng, An investigation on the development of a smart cutting tool for precision machining using SAW-based force measurement, Proc. of the 13th Euspen International Conference, Berlin, Germany (2013)

9. D. Remond, J. Mahfoudh, From transmission error measurements to angular sampling in rotating machines with discrete geometry, Shock Vib. 12, 149-161 (2005)

10. D. Remondy, Practical performances of high-speed measurement of gear transmission error or torsional vibrations with optical encoders, Meas. Sci. Technol. 9, 347-353 (1998)

11. L. Manin, G. Michon, D. Remond, R. Dufour, From transmission error measurement to pulley-belt slip determination in serpentine belt drives: Influence of tensioner and belt characteristics, Mech. Mach. Theory 44, 813-821 (2009)

12. K. Makita, M. Kagotani, H. Ueda, T. Koyama, Transmission error in synchronous belt drives with idler (influence of thickness error of belt back face under no load conditions), J. Mech. Des. 126, 148-155 (2004)

13. M. Kagotani, H. Ueda, Transmission error in synchronous belt with resonance under installation tension, J. Mech. Des. 134, 1-12 (2012)

14. UNI CEI ENV 13005:2000, Guide to the expression of uncertainty in measurement 